\title{
Use of ward closure to control outbreaks among hospitalized patients in acute care settings: a systematic review
}

\author{
H Wong ${ }^{1,2}, K_{\text {Eso }}^{1,2}$, A Ip 1,2, J Jones ${ }^{1,2}$, Y Kwon², S Powelson², J de Grood ${ }^{1,2}$, R Geransar ${ }^{1,2}$, M Santana ${ }^{1,2}$, \\ AM Joffe ${ }^{3,4}$, G Taylor ${ }^{3,4}$, B Missaghi ${ }^{2,3}$, C Pearce ${ }^{3}$, W Ghali ${ }^{1,2,3}$, J Conly 1,2,3* $^{1,2}$ \\ From 3rd International Conference on Prevention and Infection Control (ICPIC 2015) \\ Geneva, Switzerland. 16-19 June 2015
}

\section{Introduction}

Ward closure (WC) has been used as a strategy for controlling HAI outbreaks but restrict patient access, are highly disruptive and expensive.

\section{Objectives}

We conducted a systematic review to identify studies describing the impact of $\mathrm{WC}$ as an intervention for outbreak containment.

\section{Methods}

We searched MEDLINE, EMBASE, CINAHL, the Cochrane Database of Systematic Reviews, IndMED, LILACS for all years with no language restrictions plus reference lists from retrieved articles, conference proceedings and websites. Inclusion criteria were: hospitalized patients of all ages; WC as an intervention; discussion of outbreak control; and peer-reviewed. Exclusion criteria were: WC not used; full-text N/A; and studies using design or only presenting $2^{0}$ data analyses. Study quality was assessed with a Juni components approach. Six evaluative criteria were adapted from GRADE and the Downs and Black checklist to provide a measure for confidence in the estimate of effect of the body of evidence.

\section{Results}

Of 97 observational studies none included a controlled comparison between WC vs other interventions. WC was used as part of a bundle of interventions precluding a determination of its direct impact either separately or in parallel, or in sequence. We also found no universal definition of WC which was widely accepted. The included studies were of poor to moderate quality; the nature of outbreak reports rendered the use of highquality study designs and trials unfeasible.

\section{Conclusion}

With no controlled studies identified WC remains an intervention that is not evidence-based and its use can neither be actively encouraged nor discouraged. The generalizability and applicability of $\mathrm{WC}$ as a control intervention could be improved by standardizing outbreak investigation reporting.

\section{Disclosure of interest}

None declared.

\section{Authors' details}

'W21C, University of Calgary, Calgary, Alberta, Canada. ${ }^{2}$ University of Calgary, Calgary, Alberta, Canada. ${ }^{3}$ AHS, Calgary, Canada. ${ }^{4}$ University of Alberta, Edmonton, Alberta, Canada.

Published: 16 June 2015

\section{doi:10.1186/2047-2994-4-S1-053}

Cite this article as: Wong et al: Use of ward closure to control outbreaks among hospitalized patients in acute care settings: a systematic review. Antimicrobial Resistance and Infection Control 2015 4(Suppl 1):053.

${ }^{1}$ W21C, University of Calgary, Calgary, Alberta, Canada

Full list of author information is available at the end of the article 